\title{
En-face Tomographic Imaging of Scattering Objects Using Single Broadband Light Emitting Diode Based Full-Field Optical Coherence Microscopy
}

DOI: $10.17691 /$ stm2018.10.1.03

Received November 16, 2017

T. Anna, PhD, Post Doctoral Researcher ${ }^{1,2}$.

S. Chakraborty, PhD, Post Doctoral Researcher3;

A. Karmenyan, PhD, Research Fellow, Department of Physics;;

A. Chiou, PhD, Professor, Director, Biophotonics Interdisciplinary Research Center²;

W.-C. Kuo, PhD, Professor ${ }^{1,2}$

${ }^{1}$ Biophotonics and Molecular Imaging Research Center, National Yang-Ming University, 155, Sec. 2,

Li-Nong St., Taipei, 11221, Taiwan, R.O.C.;

${ }^{2}$ Institute of Biophotonics, National Yang-Ming University, 155, Sec. 2, Li-Nong St., Taipei, 11221, Taiwan, R.O.C.;

${ }^{3}$ Graduate Institute of Photonics and Optoelectronics, National Taiwan University, 1, Sec. 4, Roosevelt Rd.,

Taipei, 10617, Taiwan, R.O.C.;

${ }^{4}$ National Dong Hwa University, 1, Sec. 2, Da-Hsueh Rd., Shoufeng, Hualien, 97401, Taiwan, R.O.C.

Structural and morphological elucidation of soft scattering objects such as biological tissues is an important aspect of study for the development of diagnostic tools. Optical methods suffer from the limitation of scattering and absorption of light in such kind of objects; limiting the applicability in deep tissue characterizations. In this present work, we used a high-resolution full-field optical coherence microscopy (FFOCM) with a single broadband light emitting diode $(470-850 \mathrm{~nm})$ as illumination source for the imaging of scattering biological objects. The FF-OCM system was based on the Linnik geometry and a two-dimensional charge complementary oxide semiconductor camera (CMOS). The sequential two-dimensional spatial multiple phase-shifted interferograms were obtained by moving the sample stage using a piezoelectric transducer and the en-face FF-OCM images were subsequently reconstructed by a fast derivative-based method. The spatial resolution of the present system was 0.9 (air) and $1.38 \mu \mathrm{m}$ axially and laterally, respectively.

The present system was demonstrated for the imaging highly scattering artificial skin dressing, onion, and fish skin samples. Our results show the capability of the present system to clearly discern the structural features of the fish skin as evident from the depth intensity profiles at different positions. The present system is significantly stable, compact, and cost-effective with comparable spatial resolution compared to conventional FF-OCM systems and can be further used to distinguish a normal tissue from a diseased tissue.

Key words: full-field optical coherence microscopy; light emitting diodes; derivative-based method; scattering objects.

How to cite: Anna T., Chakraborty S., Karmenyan A., Chiou A., Kuo W.-C. En-face tomographic imaging of scattering objects using single broadband light emitting diode based full-field optical coherence microscopy. Sovremennye tehnologii v medicine 2018; 10(1): 27, https://doi.org/10.17691/stm2018.10.1.03

\section{Introduction}

Optical imaging techniques have revolutionized the biomedical research. These imaging techniques provide several advantages such as non-invasive, costefficient, fast, and pose no health side effects compared to imaging modalities using ionizing radiations. Traditional optical methods can provide micron-scale resolution at the sub-cellular and sub-organelle level at superficial depths of tissues. However, to see deep inside intact biological tissues, these light-based imaging modalities suffer from several challenges due to the high scattering and absorption of light $[1,2]$. But, statistical characteristics of scattering or attenuation coefficient of light can provide structural/morphological information at micrometer, or submicrometer resolution for disease diagnosis at the early phase [3]. Thus, imaging modalities which can explore this scattering of light as contrast for image reconstruction can provide information from deep depth of such specimens. Optical coherence tomography (OCT), as demonstrated by James Fujimoto at 1991, is such a technique and based low coherence interferometry [4]. Moreover, optical coherence microscopy (OCM) uses higher numerical aperture objectives for non-invasive, micron-scale cross-sectional depth-resolved imaging. OCT/OCM, till date, has been applied for several biomedical and nondestructive testing of materials [5].

Corresponding author: Tulsi Anna, e-mail: tulsianna@gmail.com 
Among the several types of OCT, full-field OCT/ OCM (FF-OCT/FF-OCM) can provide high-resolution images of intact tissues to characterize their structural features [6]. FF-OCM can provide $X Y$ en-face sectional images in a single exposure using two-dimensional (2D) charge coupled device [6], or charge complementary oxide semiconductor (CMOS) cameras [7]. The en-face sectional imaging is complementary to the longitudinal sectional imaging as it can provide temporal information within the en-face plane. Various FF-OCT/FF-OCM systems based on both time and frequency domain techniques for the quantitative imaging of biological samples have been developed [6-10]. In general, broadband infrared superluminescent diodes (SLDs) and white light sources based on thermal, halogen and xenon arc lamp have been used as illumination sources in FFOCM to achieve high axial resolution [10-12]. High power halogen light sources have advantages as it provides highest axial resolution in FF-OCM till date [13].

Among the several possible light sources, as mentioned above, light emitting diodes (LEDs), compact in design and cost-effective; have also been used as alternative interferometric illumination sources [14, 15]. LEDs, compared to conventional coherent light sources, provide significant advantages due to its short temporal coherence and spatial incoherence which eliminate the background and speckle noises. LEDs have been used, due to its advantages, in white light interferometry, digital holography, and vertical scanning interferometry [16-18]. A FF-OCT system with an infrared LED has been reported, providing higher resolution compared to SLD-based systems [4]. Infrared LED-based (central wavelength $\left(\lambda_{0}\right)$ $\sim 850 \mathrm{~nm}$ ) FF-OCM systems have also been used for nondestructive testing of polymer coating on pharmaceutical pellets [19], skin imaging (with multi-wavelength RGB LED) [20] etc. A FF-OCM system with nitride-based white light emitting diode (WLED) $\left(\lambda_{0} \sim 532 \mathrm{~nm}\right.$; fullwidth-half maxima $\sim 190 \mathrm{~nm}$ ) has also been reported for spectroscopic studies [21]. Till recently, Anna et al. [22] demonstrated the capability of WLED-based FF-OCM system for the characterizations of melanoma mice ear skin. WLED bears advantages over other broadband light sources such as halogen light due to its higher energy efficiency, longer lifetime, compact, less heat dissipation, and can provide high axial resolution.

In this work, we demonstrated the capability of a single broadband LED-based $\left(470-850 \mathrm{~nm}, \lambda_{0}=630 \mathrm{~nm}\right.$ ) Linnik type FF-OCM system to study highly scattering samples. Scattering can provide an endogenous contrast for the structural elucidation of materials. A CMOS camera was used to record the $2 \mathrm{D}$ spatial multiple phase-shifted interferograms at different depths of the specimen by moving the sample stage with a piezoelectric transducer (PZT). To reconstruct the FF-OCM en-face sectional images, a fast derivative-based demodulation method was used [23]. This derivative method based on discrete differences of a time corresponding to the depth sequences of interferograms; and is relatively faster in computation than the 4 or 5 phase step algorithm. The present single broadband LED-based FF-OCM system has been used, here, to image highly scattering artificial skin dressing, onion, and fish skin samples. Our results show that this scattering based method can provide structural information with high resolution and accuracy; thus promises to be used as a tool for the identification of a normal tissue from a diseased tissue.

\section{Materials and Methods}

The experimental setup of the single broadband LEDbased FF-OCM system is described in Figure 1. The detail of the setup can be found elsewhere [22]. In short, the light from single broadband LED (MBB1L3; Thorlabs, USA) $\left(470-850 \mathrm{~nm}, \lambda_{0}=630 \mathrm{~nm}\right)$ was collimated by $a$ combination of lenses $L_{1}$ and $L_{2}$ and incident into the Linnik interferometer. Two identical microscope objectives viz. $\mathrm{MO}_{1}$ and $\mathrm{MO}_{2}$ (Olympus, Japan) (10×, $\mathrm{NA}=0.3$, air) were used for the high-resolution imaging while two identical glass plates were used in sample and reference arms to compensate the dispersion mismatch and optimize axial resolution. The reference mirror was kept in focus using $\mathrm{MO}_{1}$ via a computer controlled translational stage. Sample stage was attached with a high precision PZT (NF15AP25; Thorlabs, USA) (piezoelectric translational range $25 \mu \mathrm{m}$, $25 \mathrm{~nm}$ resolution) to obtain multiple sequential phaseshifted interferograms. Back reflected light from the sample and reference mirrors interfered at the beam splitter and the interferometric signal was projected on a CMOS camera (GS3-U3-23S6M-C; Sony, Japan) (point gray, $1920(\mathrm{H}) \times 1200(\mathrm{~V})$ pixels, pixel size $5.86 \mu \mathrm{m}, \mathrm{ADC}$ 12 bits, full well pixel capacity $32,513 \mathrm{e}^{-}, 163 \mathrm{fps}$ ), by lens $\mathrm{L}_{3}$ (focal length $=60 \mathrm{~mm}$ ). All real time acquisition and image processing were done by LabView software.

The recorded intensity distribution of interferometric signal as a function of time while scanning axially through the sample can be expressed as [22, 23]:

$$
I(x, y, t)=I_{0}(x, y)+\gamma(x, y, t) A_{i}(x, y, t) \sin (\omega t)+\varphi(x, y),
$$

where $I_{0}(x, y)$ is the background intensity, $\omega=2 \pi f$ ( $f$ is the central frequency of the light source corresponds to the center wavelength of the light source i.e., $\left.\lambda_{0}\right), \varphi(x, y)$ is the phase delay due to the sample and $\gamma(x, y, t)$ is the envelope of autocorrelation of the light source. When the phase shift between two sequential images is very small compared to coherence length of the light source, then $\gamma(x, y, t) \approx 1$. However, multiple phase-shifted images were taken in a time sequence. Subsequently first, second, and third derivatives of $I(x, y, t)$ with respect to scanning time $(t)$ was expressed in terms of discrete differences of four sequential images i.e., $I_{1}(x, y), I_{2}(x, y), I_{3}(x, y)$, and $I_{4}(x, y)$. Combining these images, the en-face sectional amplitude images at $i^{\text {th }}$ layer without the requirement of the phase-shift angle can be obtained as $[22,23]$ :

$$
\begin{aligned}
& A_{i}(x, y)=\mid\left[I_{3}(x, y)-2 I_{2}(x, y)-I_{1}(x, y)\right]^{2}-\left\{I_{4}(x, y)-\right. \\
& \left.-3\left[I_{3}(x, y)-I_{2}(x, y)\right]-I_{1}(x, y)\right\}\left.\left[I_{2}(x, y)-I_{1}(x, y)\right]\right|^{1 / 2} .
\end{aligned}
$$




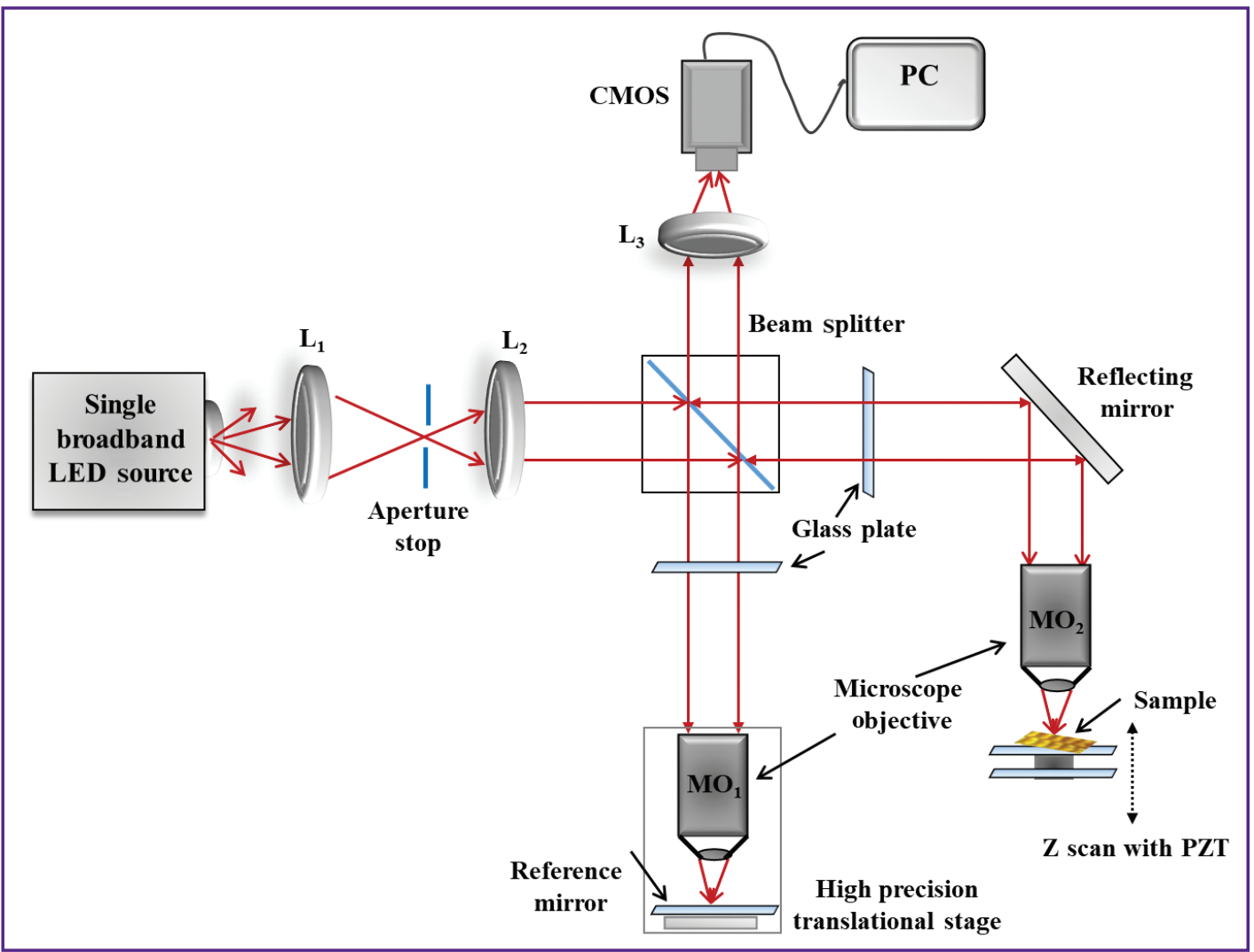

Figure 1. Schematic of the single broadband light emitting diode based full-field optical coherence microscopy system

LED: light emitting diode; $\mathrm{L}_{1}-\mathrm{L}_{3}$ : lenses; CMOS: complementary oxide semiconductor; $\mathrm{PC}$ : personal computer; PZT: piezoelectric transducer

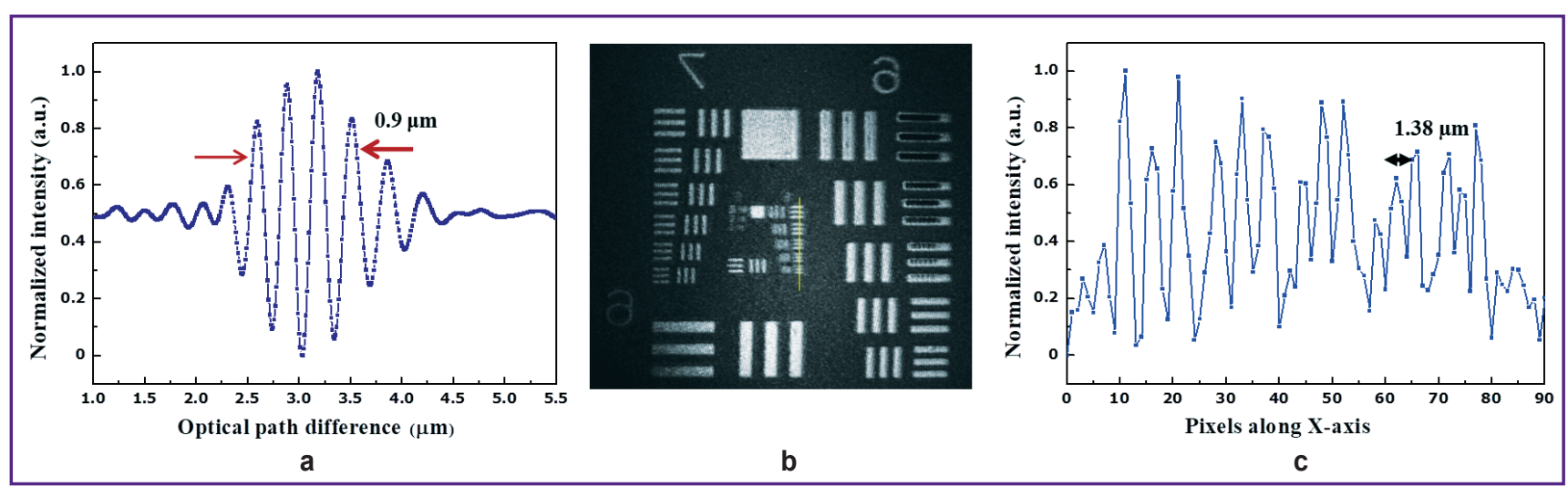

Figure 2. Characterization of the single broadband light emitting diode based full-field optical coherence microscopy system:

(a) response of the coherence function; (b) en-face OCT image of USAF 1951 test target; (c) intensity profile of the eighth group taken at the place indicated by the yellow line in (b)

The performance of the single broadband LED-based FF-OCM system for the imaging of scattering objects was evaluated by measuring the spatial (axial and lateral) resolution and signal to noise ratio (SNR). The axial resolution of the FF-OCM system is governed by the coherence length of the light source. Figure 2 (a) shows the measured coherence function as $0.9 \mu \mathrm{m}$ by using a plane reflecting mirror as a sample [22]. The axial resolution using full-width-half maxima, $\Delta \lambda=212 \mathrm{~nm}$ (measured) and refractive index $n=1$ (air) was calculated as $0.82 \mu \mathrm{m}$; thus showing both the calculated and measured axial resolutions were in good agreement with each other. In 
addition, the SNR was also measured from the obtained axial response. The measurement was performed by inserting an optical density filter $(-1.3)$ in the sample arm with a round trip attenuation of $26 \mathrm{~dB}$. A variable attenuator was used in the reference arm to keep CMOS close to the saturation level. The SNR of the system was obtained to be $-44 \mathrm{~dB}$ and limited by the full well pixel capacity of the CMOS camera. Further, the lateral resolution was also measured by reconstructing the en-face image of a standard USAF 1951 test target. The intensity profile across the lines of group 8 was plotted, and the lateral resolution of the system was obtained to be $\sim 1.38 \mu \mathrm{m}$. The calculated $(\sim 1.32 \mu \mathrm{m})$ and the measured lateral resolutions showed similar performance of the system.

We also characterized our single broadband LED- based FF-OCM system in detail by measuring the light source stability in terms of noise to signal ratio i.e., $0.50 \%$ without averaging and $0.20 \%$ with $3 \times 3$ pixel averaging at nearby saturation and phase stability in terms of standard deviation in phase i.e. $11 \mathrm{mrad}$. The detail measurement processes of these parameters also can be found in our previous research article by Anna et al. [22, 24].

\section{Results and Discussion}

Here, we imaged several scattering objects using our developed single broadband LED-based FF-OCM system such as, an artificial skin dressing, onion, and fish skins. The results of our measurements and the corresponding discussion are mentioned as follows.

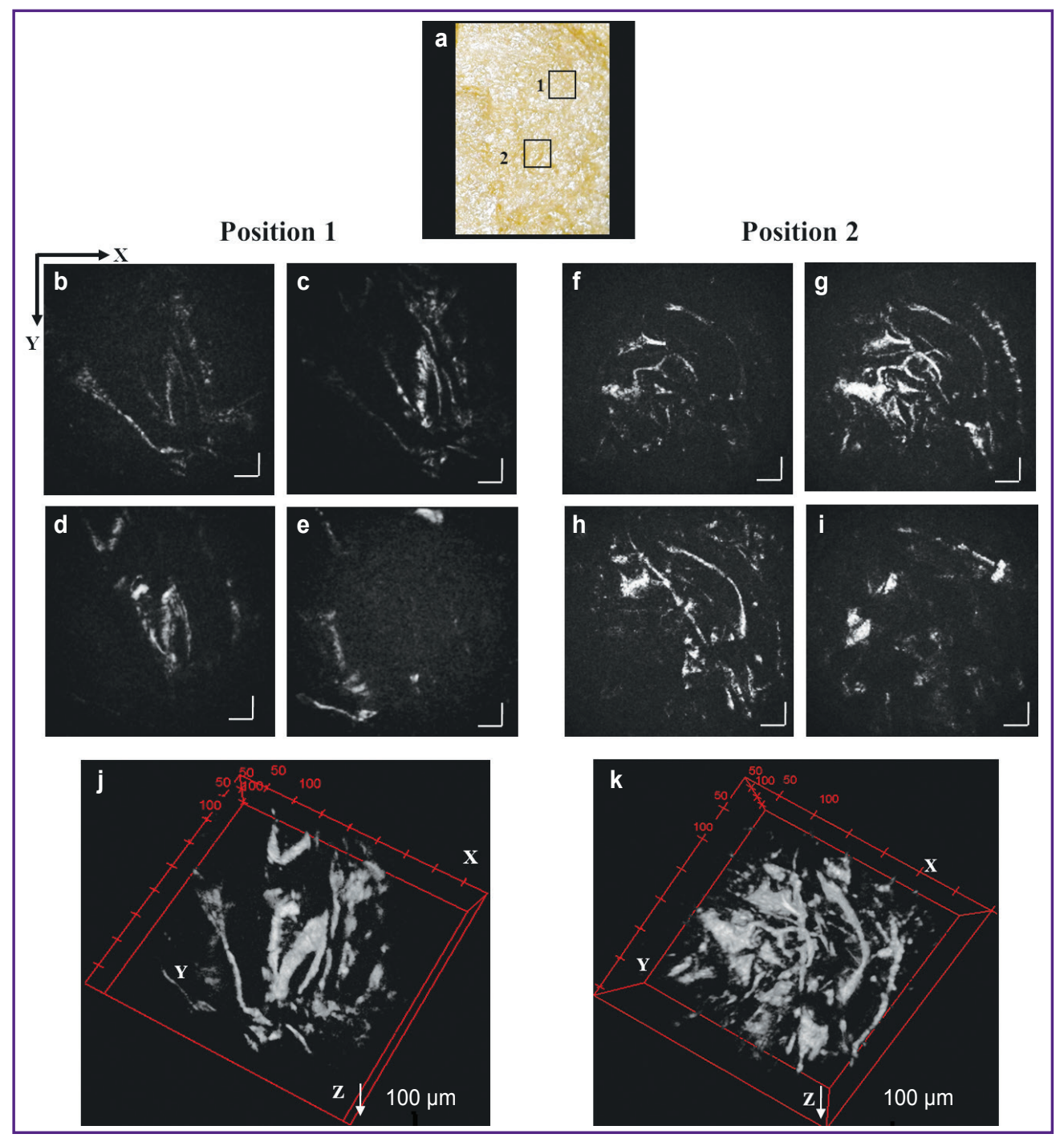

Figure 3. Single broadband-based full-field optical coherence microscopy imaging of an artificial skin dressing:

(a) photograph of skin dressing sample (indicating imaging areas; position 1 and 2); (b)-(e) en-face sectional images of skin dressing sample at 15, 38,60, and $90 \mu \mathrm{m}$ corresponds to position 1 and (f)-(i) at 15, 35, 55, and $95 \mu \mathrm{m}$ depths corresponds to position 2, respectively; (j) and (k) shows stacked 3D volume $(500 \times 500 \times 100 \mu \mathrm{m})$ images corresponding to positions 1 and 2 , respectively as marked in the photograph of the sample (a). Scale bar: $50 \mu \mathrm{m}$ 
At first, the developed FF-OCM system was used to study a highly scattering artificial skin dressing sample (Skin Temp; Taiyan Biotech Co., Ltd., China) was chosen. Skin dressings are materials which are generally used to protect wounds during healing process. These are multilayered composite structures, and can serve as an ideal scattering sample, mimicking very close to real biological tissues. The incident power of the light at the sample plane and the exposure time were $60 \mu \mathrm{W}$ and $10 \mathrm{~ms}$, respectively. The photograph of the highly scattering complex structured artificial skin dressing sample is shown in Figure 3 (a). The imaging areas are marked by position 1 and 2. The sequentially phase-shifted interferograms of the artificial skin dressing at different depths were acquired (50 fps) by moving the PZT with constant step of $33 \mathrm{~nm}$. To achieve higher depth in the sample, five sequential scans, each of $25 \mu \mathrm{m}$, by moving the PZT was performed. This was achieved by a computer controlled translation stage attached to sample stage (see Figure 1). A series of en-face FF-OCM images were then reconstructed using derivative-based method (using Eq. (2)) from the top of the sample surface to the different depths. Figure 3 (b)(e) shows the en-face FF-OCM images at 15, 38, 60, and $90 \mu \mathrm{m}$ depths for position 1 and (f)-(i), at $15,35,55$, and $95 \mu \mathrm{m}$ depths for position 2 of the sample, respectively, revealing the different complex structures of the sample. The 3D data set was obtained by combining the en-face sectional images and the 3D projection of volume $500 \times 500 \times 100 \mu \mathrm{m}$ for position 1 and 2 are shown in Figure $3(\mathrm{j})$ and $(\mathrm{k})$, respectively, discerning the layered structure of the sample.

To further evaluate the capability of imaging of scattering biological samples by the developed single broadband-based FF-OCM system, we measured the en-face images of an onion bulk and fish skin samples. The multiple phase-shifted interferograms were measured as mentioned previously and the en-face images at different depth were reconstructed. Figure 4 (a)-(c) shows the en-face FF-OCM images at 2, 20, 40 , and $64 \mu \mathrm{m}$ depths, respectively. Figure 4 (d) shows the stacked 3D volume image of $500 \times 500 \times 90 \mu \mathrm{m}$. The different cell organizations such as a cell wall inside the cell (epithelium) as well as concentric laminae layers of the onion skin are clearly resolved in these sectional images. Our approach can be implemented in studying physiological and/or pathological defects in onions and possibly other food products; thus can play an important role in food security.

We have also taken the Japanese butterfish (Psenopsis anomala) skin (Figure 5 (a)) as an example to observe the performance of the present system in elucidating structural complexity. The en-face FF-OCM images at 5,
18, 34, and $45 \mu \mathrm{m}$ depths are shown in Figure 5 (b)-(e), which reveals structural heterogeneity at different depths of epidermis layer of the skin. The scales present in the upper layers of the skin (Figure 5 (b)) and complex structure in between the scales and joints (Figure 5 (c)) are clearly visible. At 34 and $45 \mu \mathrm{m}$ depths, microridges of the epidermis can also be seen in Figure 5 (d) and (e). Figure 5 (f) shows the cross-sectional $Y Z$ view of the sample revealing the upper epidermis scale and stratum spongiosum with scale layers of epidermis structure of the fish skin. Figure $5 \mathrm{(g}$ ) shows the 3D volume image of $500 \times 500 \times 120 \mu \mathrm{m}$ depicting this structural information, which can be used for further understanding of fish skin non-invasively. The residual fringes at higher depths may be attributed to the irregular pattern of the skin surface, arising probably due phase error occurrence.

Furthermore, the depth profile along the Z-direction for the fish skin sample was also plotted for better understanding of the sample structure. The depth profile of the FF-OCM intensity for $15 \times 15$ pixels for three different positions is shown in Figure 6 corresponding to the area marked by yellow boxes in the en-face sectional image as indicated by 1, 2, and 3 in Figure 5 (c).

Figure 6 (a) and (b) shows three prominent peaks in the depth profile and some of the en-face FF-OCM images can be seen in Figure 5 (c)-(e) corresponding to first two peaks. These peaks can be attributed to fish skin epidermis scale layer and stratum spongiosum 


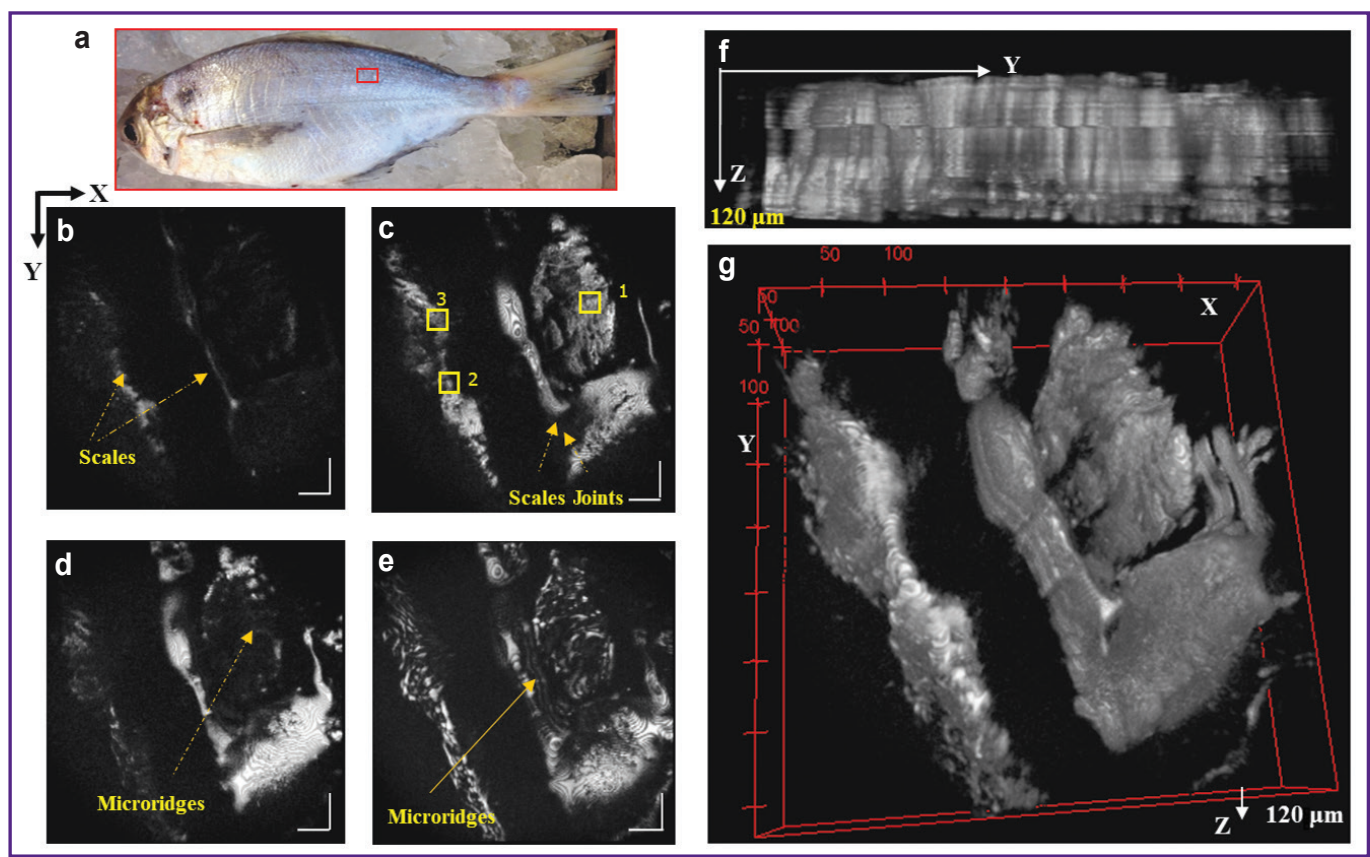

Figure 5. En-face sectional imaging of Japanese butterfish skin using single broadband-based full-field optical coherence microscopy:

(a) photograph of the fish (indicating imaging area; red rectangular box); (b)-(e) en-face sectional images of fish skin at $5,18,34$, and $45 \mu \mathrm{m}$ depths, respectively; (f) cross-sectional view along YZ; (g) stacked 3D volume image of $500 \times 500 \times 120 \mu \mathrm{m}$. Scale bar: $50 \mu \mathrm{m}$



a



b

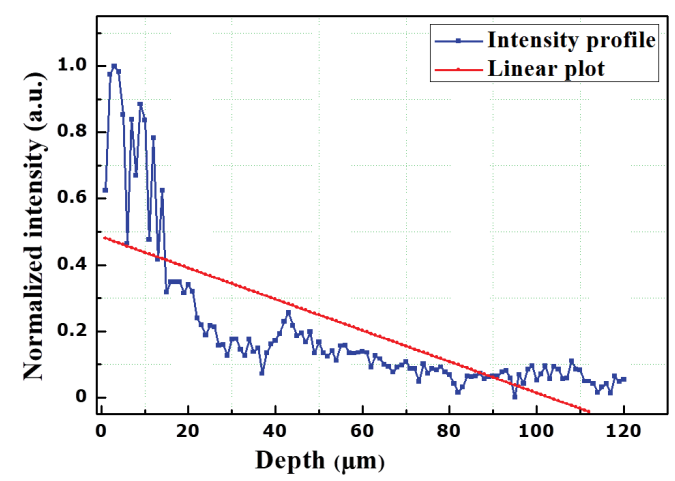

C

Figure 6. Depth intensity profiles obtained by single broadband-based full-field optical coherence microscopy:

(a)-(c) the depth profile of FF-OCM intensity corresponding to areas 1,2 , and $3(15 \times 15$ pixels) marked in the en-face images of Figure 5 (c), respectively 
region. Figure 6 (c) shows only one prominent peak indepth profile which may be attributed to the first surface of epidermis with remaining scale part. Due to the high reflectivity from this region it is not possible to see deep inside. We also applied the polyfitting to these depth intensity profiles, showing distinctly different attenuation level of incident light. The slopes of the fitted lines for the intensity profiles in Figure 6 (a)-(c) are 0.0046, 0.0047, and 0.0056, respectively. As evident from the intensity profiles and fitted slope values, it can be concluded that our FF-OCM system provides layered structural information of the fish skin. Moreover, our results indicate the high stability of the present system to measure structural features of biological samples. The present system also offers higher spatial resolutions compared to SLD-based systems and comparable with other popular single broadband light source such as halogen. In addition, single broadband LEDs provide uniform illumination of the sample, which minimizes the background noise as compared to SLD-based FF-OCM.

\section{Conclusion}

In conclusion, a single broadband LED-based FF-OCM system was used to study structural features of highly scattering samples. As examples, artificial skin dressing, onion, and fish skins were studied. Our system provides high spatial resolutions viz. $0.9 \mu \mathrm{m}$ (axial) and $1.38 \mu \mathrm{m}$ (lateral) with measured sensitivity $-44 \mathrm{~dB}$. For interferometric image reconstruction, a derivative-based method was used. Our results show the capability of the present system in elucidating the structural features of our chosen scattering samples. Intensity profiles along depth in fish skin provided information of the skin layer organization. Moreover, different cellular structures can be seen in onion skin samples. These findings confirm the robustness of the present system to further its application in human skin (dermatology) study and microstructural assessment of normal and diseased tissues.

Acknowledgments. The financial supports from Biophotonics and Molecular Imaging Research Center (BMIRC), National Yang-Ming University, Taipei, Taiwan are gratefully acknowledged.

Conflict of interest. The authors have no conflict of interests to disclose.

\section{References}

1. Ntziachristos V. Going deeper than microscopy: the optical imaging frontier in biology. Nat Methods 2010; 7(8): 603-614, https://doi.org/10.1038/nmeth.1483.

2. Badon A., Boccara A.C., Lerosey G., Fink M., Aubry A. Multiple scattering limit in optical microscopy. Opt Express 2017; 25(23): 28914-28934, https://doi.org/10.1364/ oe.25.028914.

3. Gao W. Fourier spectrum analysis of full-field optical coherence tomography for tissue imaging. Proc R Soc A 2015; 471(2179): 20150099, https://doi.org/10.1098/rspa.2015.0099.
4. Optical coherence tomography. Technology and applications. Drexler W., Fujimoto J.G. (editors). SpringerVerlag Berlin Heidelberg; 2008, https://doi.org/10.1007/978-3540-77550-8.

5. Adhi M., Duker J. S. Optical coherence tomography current and future applications. Curr Opin Ophthalmol 2013; 24(3): 213-221, https://doi.org/10.1097/icu.0b013e32835f8bf8.

6. Dubois A., Vabre L., Boccara A.C., Beaurepaire E. High-resolution full-field optical coherence tomography with a Linnik microscope. Appl Opt 2002; 41(4): 805-812, https://doi. org/10.1364/ao.41.000805.

7. Egan P., Lakestani F., Whelan M.P., Connelly M.J. Full-field optical coherence tomography with a complimentary metal-oxide semiconductor digital signal processor camera. Optical Engineering 2006; 45(1): 015601, https://doi. org/10.1117/1.2158968.

8. Anna T., Srivastava V., Mehta D.S., Shakher C. Highresolution full-field optical coherence microscopy using a Mirau interferometer for the quantitative imaging of biological cells. Appl Opt 2011; 50(34): 6343-6351, https://doi.org/10.1364/ ao.50.006343.

9. Srivastava V., Nandy S., Mehta D.S. High-resolution corneal topography and tomography of fish eye using widefield white light interference microscopy. Appl Phys Lett 2013; 102(15): 153701-153703, https://doi.org/10.1063/1.4802084.

10. Zhu Y., Gao W., Zhou Y., Guo Y., Guo F., He Y. Rapid and high-resolution imaging of human liver specimens by full-field optical coherence tomography. J Biomed Opt 2015; 20(11): 116010, https://doi.org/10.1117/1.jbo.20.11.116010.

11. Dubois A., Grieve K., Moneron G., Lecaque R., Vabre L., Boccara C. Ultrahigh-resolution full-field optical coherence tomography. Appl Opt 2004; 43(14): 2874-2883, https://doi.org/10.1364/ao.43.002874.

12. Safrani A., Abdulhalim I. Ultrahigh-resolution full-field optical coherence tomography using spatial coherence gating and quasi-monochromatic illumination. Opt Lett 2012; 37(4): 458-460, https://doi.org/10.1364/ol.37.000458.

13. Federici A., Dubois A. Full-field optical coherence microscopy with optimized ultrahigh spatial resolution. Opt Lett 2015: 40(22): 5347-5350, https://doi.org/10.1364/ ol.40.005347.

14. Dilhaire S., Grauby S., Jorez S., Lopez L.D., Rampnoux J.M., Claeys W. Surface displacement imaging by interferometry with a light emitting diode. Appl Opt 2002; 41(24): 4996-5001.

15. Warnasooriya N., Kim M.K. LED-based multiwavelength phase imaging interference microscopy. Opt Express 2007; 15(15): 9239-9247, https://doi.org/10.1364/oe.15.009239.

16. Guo R., Yao B., Min J., Zhou M., Yu X., Lei M., Yan S., Yang Y., Dan D. LED-based digital holographic microscopy with slightly off-axis interferometry. J Opt 2014; 16(12): 125408, https://doi.org/10.1088/2040-8978/16/12/125408.

17. Shavrin I., Lipiäinen L., Kokkonen K., Novotny S., Kaivola M., Ludvigsen H. Stroboscopic white-light interferometry of vibrating microstructures. Opt Express 2013; 21(14): 16901-16907, https://doi.org/10.1364/oe.21.016901.

18. Chong W.K., Li X., Soh Y. C. Harnessing spectral property of dual wavelength white LED to improve vertical scanning interferometry. Appl Opt 2013; 52(19): 4652-4662, https://doi.org/10.1364/ao.52.004652.

19. Li C., Zeitler J.A., Dong Y., Shen Y.-C. Nondestructive evaluation of polymer coating structures on pharmaceutical pellets using full-field optical coherence 
tomography. J Pharm Sci 2014; 103(1): 161-166, https://doi. org/10.1002/jps.23764.

20. Yang B.W., Chen X.C. Full-color skin imaging using RGB LED and floating lens in optical coherence tomography. Biomed Opt Express 2010, 1(5): 1341-1346, https://doi. org/10.1364/boe.1.001341.

21. Cheng H.C., Sun C.K. WLED-based low coherence interferometry in the visible wavelength range. J Med Biol Eng 2007; 27: 173-176.

22. Anna T., Chang T.-W., Lai C.-M., Chiou A., Kuo W.-C. A feasibility study of broadband white light emitting diode (WLED) based full-field optical coherence microscopy (FF-
OCM) using derivative-based algorithm. IEEE Photonics J 2017; 9(2): 3900513, https://doi.org/10.1109/jphot.2017.2686978.

23. Chang S., Cai X., Fluerau C. An efficient algorithm used for full-field optical coherence tomography. Opt Lasers Eng 2007; 45(12): 1170-1176, https://doi.org/10.1016/j. optlaseng.2007.06.008.

24. Anna T., Lai C.-M., Chiou A., Kuo W.-C. Enface sectional imaging using single-shot full-field optical coherence tomography (SS-FF-OCT) based on white light emitting diode (WLED). Proc. SPIE 10024, Optics in Health Care and Biomedical Optics VII 2016, 100243T, https://doi. org/10.1117/12.2246197. 